\section{CASE REPORT}

doi: 10.5455/medarh.2018.72.300-302

MED ARCH. 2018 AUG; 72(4): 300-302

RECEIVED: APR 04, 2018 | ACCEPTED:JUL 29, 2018

${ }^{1}$ Eye Clinic, University Clinical Centre Tuzla, Tuzla, Bosnia and Herzegovina

${ }^{2}$ Public Institution, Polyclinic with a day care hospital Doboj-Jug, Bosnia and Herzegovina

Corresponding author: Meliha Halilbasic, MD. University Clinacal Center, Eye Clinic Tuzla. Trnovac bb, 75000 Tuzla, Bosnia and Herzegovina. ORCID ID: http://www.orcid.org: Phone: +387 61728 788. E-mail: meliha1@ bih.net.ba

\title{
Congenital Bilateral Anophthalmia: a Case Report and Review of Literature
}

\author{
Meliha Halilbasic ${ }^{1}$, Vahid Jusufovic ${ }^{1}$, Zlatko Musanovic ${ }^{1}$, Arnes Cabric ${ }^{2}$
}

\section{ABSTRACT}

Introduction: Anophthalmia is congenital absence of the eyes; it may be unilateral or bilateral. Though rare, it occurs worldwide. It usually occurs in association with other systemic malformations. Case report: Therefore, the case of a female child delivered in University Clinic Center Tuzla is presented here with bilateral anophthalmia. The diagnosis was confirmed with an ocular computer tomography (CT) scan that showed under development of both globes within the orbit with a conclusion of bilateral anophthalmia. No other anomalies were found. Conclusion: This is unique case in the territory of Bosnia and Herzegovina, as according to the literature this is the first case of anophthalmia presented in this region. Currently two years ago she is followed both in ophthalmic and pediatric clinic at University Clinical Center in Tuzla. Keywords: Anophthalmia, blind child, antenatal care, reconstructive surgery.

\section{INTRODUCTION}

Anophthalmia refers to the absence of ocular tissue in the orbit and it can be unilateral or bilateral. It may occur in isolation or as part of a syndrome. It usually occurs in association with other systemic malformations particularly involving cardiac, musculoskeletal and central nervous system (1). Diagnosis of anophtlamia is usually clinical, following a complete ophthalmologic examination. This complete ophthalmological examination reveals the complete absence of the globe. Anophthalmia/microphthalmia have complex aetiology with chromosomal, monogenic and environmental causes identified. Chromosomal duplications, deletions and translocations are implicated. Of monogenic causes only SOX2 has been identified as a major causative gene (1).

The birth prevalence of anophthalmia and microphthalmia has been generally estimated to be 3 and 14 per 100,000 population respectively $(2,3)$. In Sweden, a prevalence of 0.2-0.4 per 10,000 live births was reported (4). In Benin city Nigeria, authors (5) reported two cases seen in a period of 20 years. In Enugu, Southeastern Nigeria, anophthalmia represented $9.3 \%$ of congenital eye anomalies in a period of 8 year (6). In United Kingdom, the overall prevalence of anopthalmia and microthalmia was 1.0 per 10000 births (7). In Northeast Italy during the period from 1981 to 1989,22 cases were detected among 368,256 births yielding a birth prevalence of 0.60 per 10,000 (8). This is the first reported case of anophthalmia in Bosnia and Herzegovina.

We, report a case of a female child who was delivered with bilateral anophthalmia, that occurred in isolation without any kind of syndrome. After the complete examination non cardiac, musculoskeletal or central nervous system deformation were found.

\section{CASE REPORT}

Ophthalmologist was called for consultation at University Clinic Center Tuzla, Clinic of Gynecology and Obstetrics to see a newborn female baby. It was reported to the ophthalmolohist that after 24 hours from the birth, female newborn has not opened eyes and that there was a livid swelling of a both lower eye lid-resembling hemorrhagic cysts. The infant was born by normal spontaneous vaginal delivery to a 21 years old mother following a full term pregnancy. The mother had antenatal care in local health center "Teočak". According to the mother pregnancy went without any kind of complications. This was a second pregnancy from healthy parents. Mother reported that she has lost her first pregnancy, but didn't report the reason. There was no history of congenital infections like toxoplasma or rubella or any significant teratogenic drug intake during pregnancy. No history of fever, body rashes and no exposure to $\mathrm{X}$-rays. She also took 


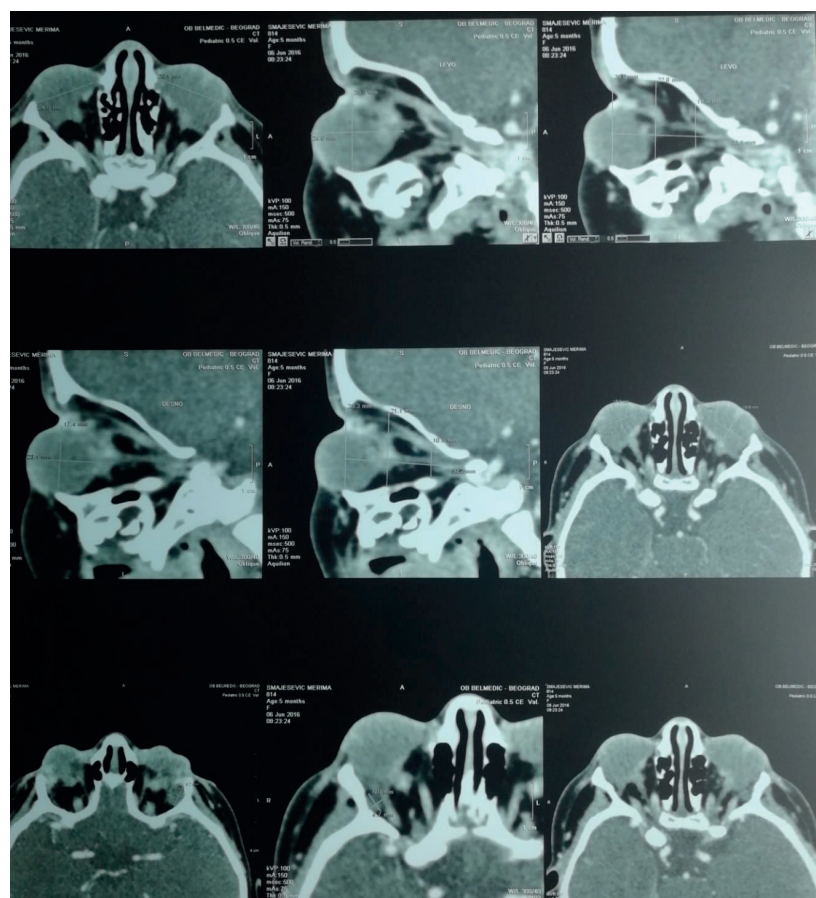

Figure 1. Brain CT examination at our patient with anophthalmia

prenatal vitamins but denied any other medication, illicit drug, alcohol, or tobacco exposure. There was no family history of anophthalmia/ microphthalmia or significant neurological anomalies. On the examination baby had a normal weight of 3440 grams, during ophthalmological examination we found deeply set orbits, small palpebral fissures and livid hemorrhagic cyst $\mathrm{s}$ on both lower lids. The eyeball could not be felt on palpation. There was no associated dysmorphic facies or other neurological abnormalities. The auditory and motor system examinations were normal. The diagnosis was confirmed with an ocular computer tomography (CT) scan that showed under development of both globes within the orbit with a conclusion of bilateral anophthalmia; a brain CT was normal (Figure 1). Chest X-ray and abdominal ultrasound scan were normal. An echocardiography was also normal. MRI of brain showed no pathological changes and MRI of both orbit in expected location of the globes showed irregular ovoid structures without developed intraocular lens, that could resemble dysplastic globes. Optic nerve was present. Antibodies for Toxoplasma, Rubella and Cytomegalovirus were negative. As the case of bilateral anophthalmia was confirmed. Child was sent to Private Hospital in Belgrade for a further evaluation and possible surgery (Figure 2). After the evaluation done in Belgrade, surgery was performed and further prosthetic treatment was done.(Figure 3.)

\section{DISCUSSION}

Our patient presented with bilateral anophthalmia. The defect is often bilateral, similar to that in our patient. Reviewing literature we couldn't find any case of anophthalmia in Bosnia and Herzegovina prior to this case. Although anophthalmia is closely linked to genetic and familiar causes, our patient had no family history of similar condition (1). Our patient's condition was diagnosed

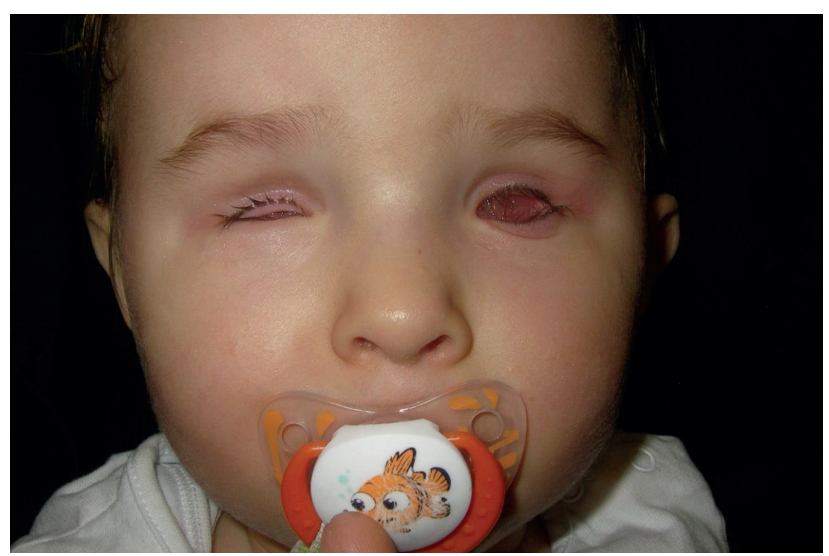

Figure 2. Patient with anophthalmia before surgical treatment

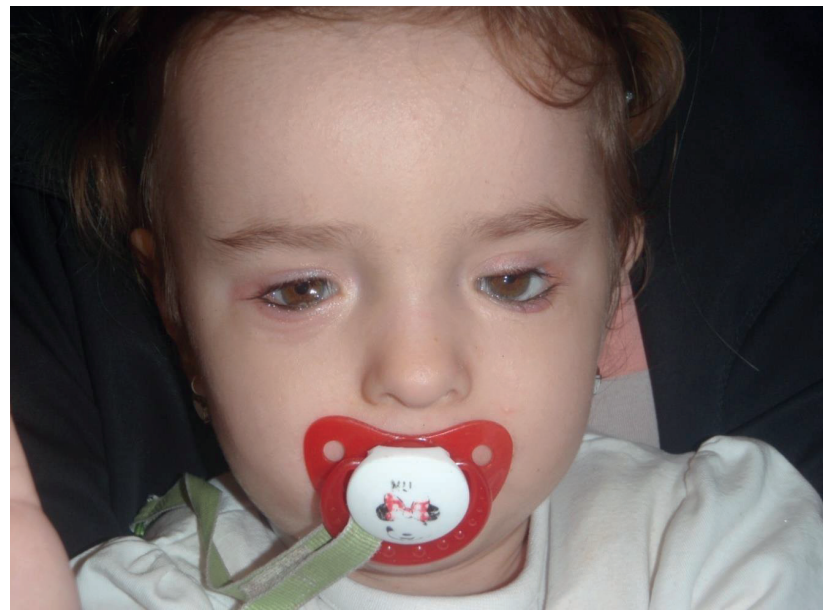

Figure 3. Patient after prosthetic surgical treatment at Private hospital in Belgrade, Serbia

after birth and antenatal ultrasonography failed to pick the anomaly. Possible reason for not picking the anomaly antenatal is the old equipment used in rural health care centers. Ultrasound has been a very important tool in the prenatal diagnosis of malformations, especially after the improvement in the quality of the images. Anophthalmia diagnosis can be made by two-dimensional (2D) ultrasonography when eyeballs and lens are absent, but using three-dimensional (3D) ultrasonography we have more information, especially when the fetal head position is unfavorable. This way we want to emphasize the importance of fetal screening during pregnancy in developing countries such as Bosnia and Herzegovina. CT and MR scans facilitate the diagnosis of anophthalmia. Both scans show the absence of a globe within the orbit although soft amorphous tissue may be seen. It is also important to emphasize that ophthalmologist in this kind of patient is the primary doctor of the patient. Also it is important to mention that the job of ophthalmologist is to coordinate a multidisciplinary team as the patient could have optimal care.

- Declaration of patient consent: Authors certify that they have obtained patient consent form.

- Conflict of interest: none declared. 


\section{REFERENCES}

1. Verma AS, Fitzpatrick DR. Anophthalmia and microphthalmia. Orphanet J Rare Dis. 2007; 2: 47.

2. Morrison D, FitzPatrick D, Hanson I, Williamson K, van Heyningen V, Fleck B, et al. National study of microphthalmia, anophthalmia, and coloboma (MAC) in Scotland: Investigation of genetic aetiology. J Med Genet. 2002; 39: 16-22.

3. Campbell H, Holmes E, MacDonald S, Morrison D, Jones I. A capturerecapture model to estimate prevalence of children born in Scotland with developmental eye defects. J Cancer Epidemiol Prev. 2002; 7: 2128.

4. Källén B, Tornqvist K. The epidemiology of anophthalmia and microphthalmia in Sweden. Eur J Epidemiol. 2005; $20: 345350$.

5. Ukponmwan CU. Congenital anophthalmos in Benin City,
Nigeria. West Afr J Med. 1999; 18: 141143

6. ChukaOkosa CM, Magulike NO, Onyekonwu GC. Congenital eye anomalies in Enugu, SouthEastern Nigeria. West Afr J Med. 2005; 24: 112114.

7. Dolk H, Cuzick J, Busby A. Armstrong BG, Walls PH. Geographical varation in anophthalmia and microphthalmia in England. 1988-94 Commentary: Clustering of anophthalmia and microophthalmnia is not supported by the data. BMJ. 1998: 317: 905.

8. H.S. Wong S, Parker J, Tait KC. PringleAntenatal diagnosis of anophthalmia by three-dimensional ultrasound: a novel. application of the reverse face view Ultrasound Obstet Gynecol. 2008; 32: 103-105.

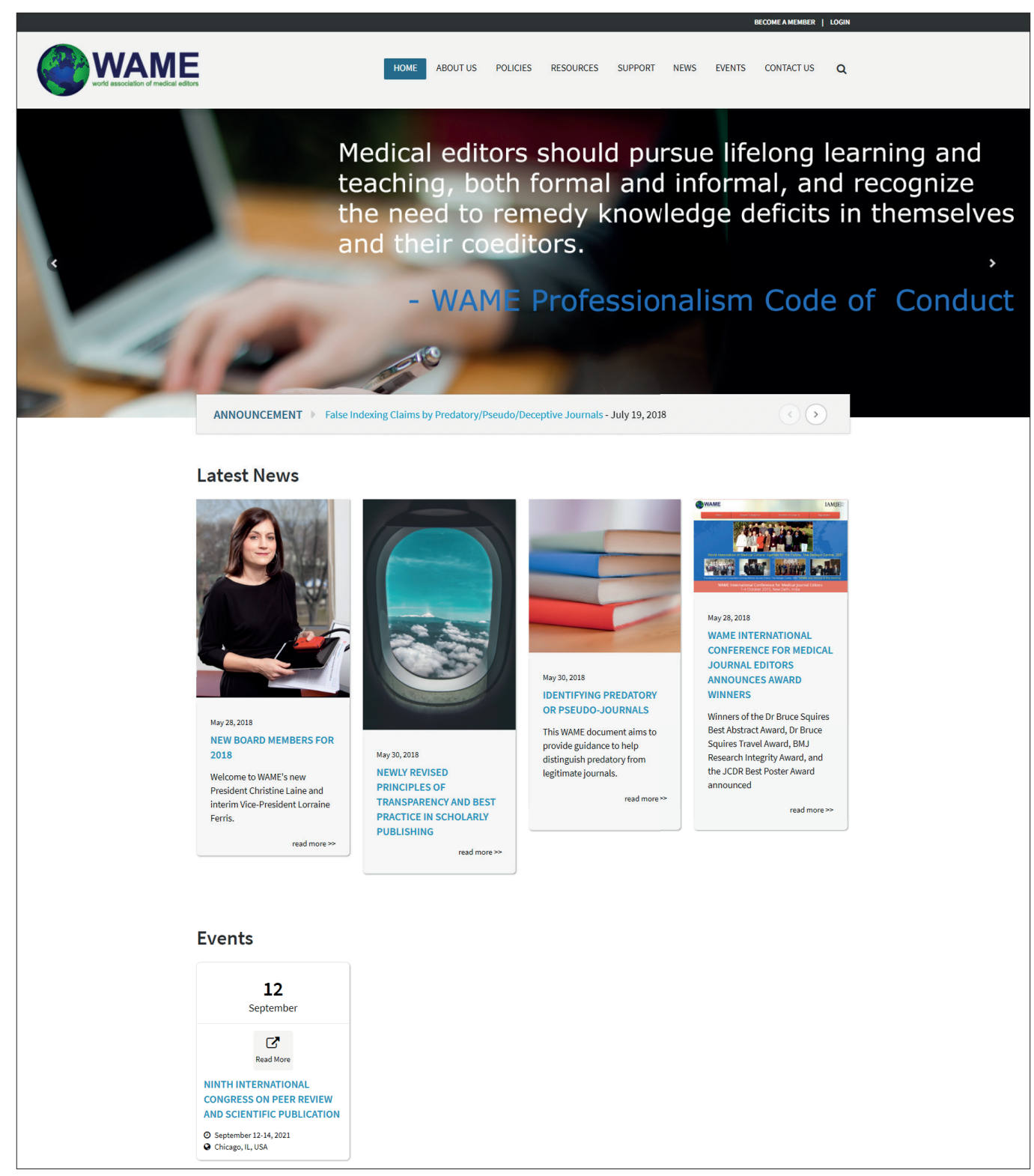

